\title{
Monotone iterative solutions for a coupled system of $p$-Laplacian differential equations involving the Riemann-Liouville fractional derivative
}

$\mathrm{Bo} \mathrm{Bi}^{1,2}$ and Ying $\mathrm{He}^{2^{*}}$

\section{"Correspondence:}

heying65332015@163.com ${ }^{2}$ School of Mathematics and Statistics, Northeast Petroleum University, Daqing 163318, P.R. China

Full list of author information is available at the end of the article

\section{Springer}

\begin{abstract}
Applying the monotone iterative technique and the method of upper and lower solutions, we investigate the existence of extremal solutions for a nonlinear system of $p$-Laplacian differential equations with nonlocal coupled integral boundary conditions. We present a numerical example to illustrate the main result.
\end{abstract}

MSC: $34 \mathrm{~B} 15$

Keywords: Fractional differential system; Nonlocal coupled integral boundary conditions; Extremal solution; $p$-Laplacian operator; Monotone iterative technique

\section{Introduction}

Consider the following fractional differential system with the nonlocal coupled integral boundary conditions:

$$
\left\{\begin{aligned}
-D^{\beta}\left(\phi_{p}\left(-D^{\alpha} x(t)\right)\right) & =f\left(t, x(t), y(t), D^{\alpha} x(t), D^{\alpha} y(t)\right), \quad t \in(0,1], \\
-D^{\beta}\left(\phi_{p}\left(-D^{\alpha} y(t)\right)\right) & \left.=g\left(t, y(t), x(t), D^{\alpha} y(t)\right), D^{\alpha} x(t)\right), \quad t \in(0,1], \\
D^{\alpha} x(0)=0, & \quad=\frac{1}{\Gamma(\sigma)} \int_{0}^{\eta}(\eta-s)^{\sigma-1} h\left(s, \phi_{p}\left(-D^{\alpha} x(s)\right)\right) d s+a_{1}, \\
D^{\beta-1}\left(\phi_{p}\left(-D^{\alpha} x(1)\right)\right) & =I^{\sigma} h\left(\eta, \phi_{p}\left(-D^{\alpha} x(\eta)\right)\right)+a_{1} \\
x(0)=0, \quad D^{\alpha-1} x(1)=I^{\omega} x(\xi)+d_{1}=\frac{1}{\Gamma(\omega)} \int_{0}^{\xi}(\xi-s)^{\omega-1} x(s) d s+d_{1}, & \\
D^{\alpha} y(0)=0, \quad & =\frac{1}{\Gamma(\sigma)} \int_{0}^{\eta}(\eta-s)^{\sigma-1} k\left(s, \phi_{p}\left(-D^{\alpha} y(s)\right)\right) d s+a_{2}, \\
D^{\beta-1}\left(\phi_{p}\left(-D^{\alpha} y(1)\right)\right) & =I^{\sigma} k\left(\eta, \phi_{p}\left(-D^{\alpha} y(\eta)\right)+a_{2}\right.
\end{aligned}\right.
$$

where $D^{\alpha}$ and $D^{\beta}$ are the standard Riemann-Liouville fractional derivatives, $I^{\sigma}$ and $I^{\omega}$ are the Riemann-Liouville fractional integrals, and $1<\alpha, \beta<2, \sigma, \omega>1,0<\eta, \xi<1$,

(c) The Author(s) 2021. This article is licensed under a Creative Commons Attribution 4.0 International License, which permits use, sharing, adaptation, distribution and reproduction in any medium or format, as long as you give appropriate credit to the original author(s) and the source, provide a link to the Creative Commons licence, and indicate if changes were made. The images or other third party material in this article are included in the article's Creative Commons licence, unless indicated otherwise in a credit line to the material. If material is not included in the article's Creative Commons licence and your intended use is not permitted by statutory regulation or exceeds the permitted use, you will need to obtain permission directly from the copyright holder. To view a copy of this licence, visit http://creativecommons.org/licenses/by/4.0/ 
$a_{1}, a_{2}, d_{1}, d_{2} \in \mathbb{R}, a_{2} \geq a_{1}, d_{2} \geq d_{1}, f, g \in C([0,1] \times \mathbb{R} \times \mathbb{R}, \mathbb{R}), h, k \in C([0,1] \times \mathbb{R}, \mathbb{R})$. The $p$-Laplacian operator is defined as $\phi_{p}(t)=|t|^{p-2} t, p>1$, and $\left(\phi_{p}\right)^{-1}=\phi_{q}, \frac{1}{p}+\frac{1}{q}=1$.

Fractional differential equations have recently gained much attention. In particular, much effort has been made toward the study of the existence of solutions for fractional differential equations with $p$-Laplacian operator [1-8]. The monotone iterative technique, combined with the method of upper and lower solutions, is a powerful tool for proving the existence of solutions of nonlinear differential equations; see $[9,10]$ and the references therein. However, only a few papers considered the upper an lower solutions method and the monotone iteration technique for $p$-Laplacian boundary value problems with fractional coupled systems. The purpose of this paper is developing a monotone iterative technique to show the existence of an extremal solution for the nonlinear system (1.1) with nonlocal integral boundary conditions.

The paper is organized as follows. In Sect. 2, we give sufficient conditions guaranteeing that (1.1) has an extremal solution and discuss some comparison results, which play a key role in establishing the proposed work. In Sect. 3, we give the main result. Finally, we present an example illustrating our results.

\section{Preliminaries}

In this section, we introduce definitions and some useful lemmas which play an important role in obtaining the main results of this paper.

Denote

$$
C_{\alpha}[0,1]=\left\{u: u \in C[0,1], D^{\alpha} u(t) \in C[0,1]\right\} .
$$

It is a Banach spaces with the norm $\|u\|_{\alpha}=\|u\|+\left\|D^{\alpha} u\right\|$, where $\|u\|=\max _{0 \leq t \leq 1}|u(t)|$ and $\left\|D^{\alpha} u\right\|=\max _{0 \leq t \leq 1}\left|D^{\alpha} u(t)\right|$.

We need the following assumptions.

$\left(H_{1}\right)$ There exist $x_{0}, y_{0} \in C_{\alpha}[0,1]$ satisfying $D^{\beta}\left(\phi_{p}\left(-D^{\alpha} x_{0}(t)\right)\right), D^{\beta}\left(\phi_{p}\left(-D^{\alpha} y_{0}(t)\right)\right) \in C[0,1]$, $x_{0}(t) \leq y_{0}(t)$, and $D^{\alpha} y_{0}(t) \leq D^{\alpha} x_{0}(t)$ such that

$$
\left\{\begin{array}{l}
-D^{\beta}\left(\phi_{p}\left(-D^{\alpha} x_{0}(t)\right)\right) \leq f\left(t, x_{0}(t), y_{0}(t), D^{\alpha} x_{0}(t), D^{\alpha} y_{0}(t)\right), \quad t \in(0,1], \\
D^{\alpha} x_{0}(0)=0, \quad D^{\beta-1}\left(\phi_{p}\left(-D^{\alpha} x_{0}(1)\right)\right) \leq I^{\sigma} h\left(\eta, \phi_{p}\left(-D^{\alpha} x_{0}(\eta)\right)\right)+a_{1}, \\
x_{0}(0)=0, \quad D^{\alpha-1} x_{0}(1) \leq I^{\omega} x_{0}(\xi)+d_{1}, \\
\left.-D^{\beta}\left(\phi_{p}\left(-D^{\alpha} y_{0}(t)\right)\right) \geq g\left(t, y_{0}(t), x_{0}(t), D^{\alpha} y_{0}(t)\right), D^{\alpha} x_{0}(t)\right), \quad t \in(0,1], \\
D^{\alpha} y_{0}(0)=0, \quad D^{\beta-1}\left(\phi_{p}\left(-D^{\alpha} y_{0}(1)\right)\right) \geq I^{\sigma} k\left(\eta, \phi_{p}\left(-D^{\alpha} y_{0}(\eta)\right)\right)+a_{2}, \\
y_{0}(0)=0, \quad D^{\alpha-1} y_{0}(1) \geq I^{\omega} y_{0}(\xi)+d_{2} .
\end{array}\right.
$$

$\left(H_{2}\right)$ There exist two constants $M, N \in \mathbb{R}, M \geq N$, such that

$$
\begin{aligned}
& f\left(t, \overline{x(t)}, \overline{y(t)}, D^{\alpha} \overline{x(t)}, D^{\alpha} \overline{y(t)}\right)-f\left(t, x(t), y(t), D^{\alpha} x(t), D^{\alpha} y(t)\right) \\
& \quad \leq M\left[\phi_{p}\left(-D^{\alpha} x(t)\right)-\phi_{p}\left(-D^{\alpha} \overline{x(t)}\right)\right]+N\left[\phi_{p}\left(-D^{\alpha} y(t)\right)-\phi_{p}\left(-D^{\alpha} \overline{y(t)}\right)\right] \\
& g\left(t, \overline{x(t)}, \overline{y(t)}, D^{\alpha} \overline{x(t)}, D^{\alpha} \overline{y(t)}\right)-g\left(t, x(t), y(t), D^{\alpha} x(t), D^{\alpha} y(t)\right) \\
& \quad \leq M\left[\phi_{p}\left(-D^{\alpha} x(t)\right)-\phi_{p}\left(-D^{\alpha} \overline{x(t)}\right)\right]+N\left[\phi_{p}\left(-D^{\alpha} y(t)\right)-\phi_{p}\left(-D^{\alpha} \overline{y(t)}\right)\right],
\end{aligned}
$$


where $x_{0}(t) \leq \overline{x(t)} \leq x(t) \leq y_{0}(t), x_{0}(t) \leq y(t) \leq \overline{y(t)} \leq y_{0}(t)$, and

$$
\begin{aligned}
& f\left(t, x(t), y(t), D^{\alpha} x(t), D^{\alpha} y(t)\right)-g\left(t, y(t), x(t), D^{\alpha} y(t), D^{\alpha} x(t)\right) \\
& \quad \leq M\left[\phi_{p}\left(-D^{\alpha} y(t)\right)-\phi_{p}\left(-D^{\alpha} x(t)\right)\right]+N\left[\phi_{p}\left(-D^{\alpha} x(t)\right)-\phi_{p}\left(-D^{\alpha} y(t)\right)\right]
\end{aligned}
$$

for $x_{0}(t) \leq x(t) \leq y(t) \leq y_{0}(t)$.

$\left(H_{3}\right)$ There exists a constant $\lambda \geq 0$ such that

$$
\begin{aligned}
& h\left(t, \phi_{p}\left(-D^{\alpha} y(t)\right)\right)-h\left(t, \phi_{p}\left(-D^{\alpha} x(t)\right)\right) \geq \lambda\left[\phi_{p}\left(-D^{\alpha} y(t)\right)-\phi_{p}\left(-D^{\alpha} x(t)\right)\right], \\
& k\left(t, \phi_{p}\left(-D^{\alpha} y(t)\right)\right)-k\left(t, \phi_{p}\left(-D^{\alpha} x(t)\right)\right) \geq \lambda\left[\phi_{p}\left(-D^{\alpha} y(t)\right)-\phi_{p}\left(-D^{\alpha} x(t)\right)\right],
\end{aligned}
$$

where $x_{0}(t) \leq x(t) \leq y(t) \leq y_{0}(t), D^{\alpha} y_{0}(t) \leq D^{\alpha} y(t) \leq D^{\alpha} x(t) \leq D^{\alpha} x_{0}(t), t \in[0,1]$, and

$$
k\left(t, \phi_{p}\left(-D^{\alpha} y(t)\right)\right)-h\left(t, \phi_{p}\left(-D^{\alpha} x(t)\right)\right) \geq \lambda\left[\phi_{p}\left(-D^{\alpha} y(t)\right)-\phi_{p}\left(-D^{\alpha} x(t)\right)\right]
$$

for $x_{0}(t) \leq x(t) \leq y(t) \leq y_{0}(t), D^{\alpha} y_{0}(t) \leq D^{\alpha} y(t) \leq D^{\alpha} x(t) \leq D^{\alpha} x_{0}(t), t \in[0,1]$.

$\left(H_{4}\right) \Gamma(\beta+\sigma)>\lambda \eta^{\beta+\sigma-1}$.

$\left(H_{5}\right) 2 \Gamma(\beta+\sigma)(M+N)<\Gamma(\beta)\left[\Gamma(\beta+\sigma)-\lambda \eta^{\beta+\sigma-1}\right]$.

$\left(H_{6}\right)$ For any $t \in(0,1)$, we have

$$
\Gamma(2-\beta) \lambda \eta^{\sigma}<\Gamma(\sigma) .
$$

Lemma 2.1 ([11]) Let $h \in C[0,1], b \in \mathbb{R}$, and $\Gamma(\beta+\sigma) \neq \lambda \eta^{\beta+\sigma-1}$. Then the fractional boundary value problem

$$
\left\{\begin{array}{l}
-D^{\beta} w(t)=h(t), \quad t \in[0,1] \\
w(0)=0 \\
D^{\beta-1} w(1)=\lambda I^{\sigma} w(\eta)+b=\frac{\lambda}{\Gamma(\sigma)} \int_{0}^{\eta}(\eta-s)^{\sigma-1} w(s) d s+b
\end{array}\right.
$$

has the following integral representation of the solution:

$$
w(t)=\int_{0}^{1} G(t, s) h(s) d s+\frac{b \Gamma(\beta+\sigma) t^{\beta-1}}{\Gamma(\beta)\left[\Gamma(\beta+\sigma)-\lambda \eta^{\beta+\sigma-1}\right]},
$$

where

$$
G(t, s)=\frac{1}{\Delta} \begin{cases}{\left[\Gamma(\beta+\sigma)-\lambda(\eta-s)^{\beta+\sigma-1}\right] t^{\beta-1}} & \\ \quad-\left[\Gamma(\beta+\sigma)-\lambda \eta^{\beta+\sigma-1}\right](t-s)^{\beta-1}, & t \leq s \leq \eta, \eta, \\ \Gamma(\beta+\sigma) t^{\beta-1}-\lambda(\eta-s)^{\beta+\sigma-1} t^{\beta-1}, & \\ \Gamma(\beta+\sigma)\left[t^{\beta-1}-(t-s)^{\beta-1}\right]+\lambda \eta^{\beta+\sigma-1}(t-s)^{\beta-1}, & \eta \leq s \leq t, \\ \Gamma(\beta+\sigma) t^{\beta-1}, & s \geq t, s \geq \eta,\end{cases}
$$

and $\Delta=\Gamma(\beta)\left[\Gamma(\beta+\sigma)-\lambda \eta^{\beta+\sigma-1}\right]$ 
Lemma $2.2([11])$ Let $M, b \in \mathbb{R}, h(t) \in C[0,1], 2 \Gamma(\beta+\sigma)|M|<\Gamma(\beta)\left[\Gamma(\beta+\sigma)-\lambda \eta^{\beta+\sigma-1}\right]$, and $\left(H_{4}\right)$ hold. Then

$$
\left\{\begin{array}{l}
-D^{\beta} w(t)+M w(t)=h(t), \quad t \in[0,1] \\
w(0)=0 \\
D^{\beta-1} w(1)=\lambda I^{\sigma} w(\eta)+b
\end{array}\right.
$$

has a unique solution $w(t) \in C[0,1]$.

Lemma 2.3 ([10, Lemma 2.4]) Let $z(t) \in C[0,1]$ and $l \in \mathbb{R}$. Then the fractional value boundary problem

$$
\left\{\begin{array}{l}
-D^{\alpha} u(t)=z(t), \quad 0<t<1, \\
u(0)=0, \quad D^{\alpha-1} u(1)=l
\end{array}\right.
$$

is equivalent to

$$
u(t)=\int_{0}^{1} H(t, s) z(s) d s+\frac{l t^{\alpha-1}}{\Gamma(\alpha)}
$$

where

$$
H(t, s)= \begin{cases}t^{\alpha-1}-(t-s)^{\alpha-1}, & 0 \leq s \leq t \leq 1 \\ t^{\alpha-1}, & 0 \leq t \leq s \leq 1\end{cases}
$$

Lemma 2.4 Assume that $1<\alpha, \beta<2, \sigma_{1}, \sigma_{2} \in C[0,1], M, N$ are nonnegative constants satisfying $M \geq N$, and $\left(H_{4}\right)$ and $\left(H_{5}\right)$ hold. Then the fractional differential system

$$
\begin{cases}-D^{\beta}\left(\phi_{p}\left(-D^{\alpha} x(t)\right)\right)=\sigma_{1}(t)-M\left(\phi_{p}\left(-D^{\alpha} x(t)\right)\right)-N\left(\phi_{p}\left(-D^{\alpha} y(t)\right)\right), & t \in(0,1], \\ -D^{\beta}\left(\phi_{p}\left(-D^{\alpha} y(t)\right)\right)=\sigma_{2}(t)-M\left(\phi_{p}\left(-D^{\alpha} y(t)\right)\right)-N\left(\phi_{p}\left(-D^{\alpha} x(t)\right)\right), & t \in(0,1], \\ D^{\alpha} x(0)=0, \quad D^{\beta-1}\left(\phi_{p}\left(-D^{\alpha} x(1)\right)\right)=\lambda I^{\sigma} \phi_{p}\left(-D^{\alpha} x(\eta)\right)+b_{1}, & \\ x(0)=0, & D^{\alpha-1} x(1)=l_{1}, \\ D^{\alpha} y(0)=0, & D^{\beta-1}\left(\phi_{p}\left(-D^{\alpha} y(1)\right)\right)=\lambda I^{\sigma} \phi_{p}\left(-D^{\alpha} y(\eta)\right)+b_{2}, \\ y(0)=0, & D^{\alpha-1} y(1)=l_{2},\end{cases}
$$

has a unique solution in $C_{\alpha}[0, T] \times C_{\alpha}[0, T]$.

Proof Let

$$
\phi_{p}\left(-D^{\alpha} x(t)\right)=\frac{u(t)+v(t)}{2} \quad \text { and } \quad \phi_{p}\left(-D^{\alpha} y(t)\right)=\frac{u(t)-v(t)}{2}, \quad \forall t \in[0,1] .
$$

Using (2.3), we have that

$$
\left\{\begin{array}{l}
-D^{\beta} u(t)=\sigma_{1}(t)+\sigma_{2}(t)-(M+N) u(t), \\
u(0)=0, \\
D^{\beta-1} u(1)=\lambda I^{\sigma} u(\eta)+b_{1}+b_{2},
\end{array}\right.
$$


and

$$
\left\{\begin{array}{l}
-D^{\beta} v(t)=\sigma_{1}(t)-\sigma_{2}(t)-(M-N) v(t) \\
u(0)=0 \\
D^{\beta-1} v(1)=\lambda I^{\sigma} v(\eta)+b_{1}-b_{2} .
\end{array}\right.
$$

Since $M, N$ are nonnegative constants and $M \geq N$, by assumption $\left(H_{5}\right)$ we see that

$$
2 \Gamma(\beta+\sigma)(M-N) \leq 2 \Gamma(\beta+\sigma)(M+N)<\Gamma(\beta)\left[\Gamma(\beta+\sigma)-\lambda \eta^{\beta+\sigma-1}\right] .
$$

By (2.6) and Lemma 2.2 we know that (2.4) and (2.5) have a unique solution. In consequence, $\phi_{p}\left(-D^{\alpha} x(t)\right)$ and $\left.\phi_{p}\left(-D^{\alpha} y(t)\right)\right)$ are also unique, that is,

$$
\phi_{p}\left(-D^{\alpha} x(t)\right)=\omega_{1}(t) \in C[0,1], \quad \phi_{p}\left(-D^{\alpha} y(t)\right)=\omega_{2}(t) \in C[0,1] .
$$

Then

$$
\left.-D^{\alpha} x(t)=\phi_{q}\left(\omega_{1}(t)\right), \quad-D^{\alpha} y(t)\right)=\phi_{q}\left(\omega_{2}(t)\right) .
$$

In view of the boundary value condition (2.3), we obtain

$$
\left\{\begin{array}{l}
-D^{\alpha} x(t)=\phi_{q}\left(\omega_{1}(t)\right), \\
\left.-D^{\alpha} y(t)\right)=\phi_{q}\left(\omega_{2}(t)\right), \\
x(0)=0, \quad D^{\alpha-1} x(1)=l_{1}, \\
y(0)=0, \quad D^{\alpha-1} y(1)=l_{2} .
\end{array}\right.
$$

Let

$$
x(t)=\frac{p(t)+q(t)}{2} \quad \text { and } \quad y(t)=\frac{p(t)-q(t)}{2} .
$$

Using (2.7), we have

$$
\left\{\begin{array}{l}
-D^{\alpha} p(t)=\phi_{q}\left(\omega_{1}(t)\right)+\phi_{q}\left(\omega_{2}(t)\right) \\
p(0)=0 \\
D^{\alpha-1} p(1)=l_{1}+l_{2}
\end{array}\right.
$$

and

$$
\left\{\begin{array}{l}
-D^{\alpha} q(t)=\phi_{q}\left(\omega_{1}(t)\right)-\phi_{q}\left(\omega_{2}(t)\right) \\
q(0)=0 \\
D^{\alpha-1} q(1)=l_{1}-l_{2}
\end{array}\right.
$$

By Lemma 2.3 we know that both (2.8) and (2.9) have a unique solution. In consequence, $x$ and $y$ are also unique. 
Lemma 2.5 ([9, Lemma 2.6]) Let $M$ be nonnegative constant, and let $\left(H_{6}\right)$ hold. If $w(t) \in$ $C[0,1]$ satisfies $D^{\beta} w(t) \in C[0,1]$ and

$$
\left\{\begin{array}{l}
-D^{\beta} w(t) \geq-M w(t), \quad t \in[0,1] \\
w(0)=0 \\
D^{\beta-1} w(1) \geq \lambda I^{\sigma} w(\eta),
\end{array}\right.
$$

then $w(t) \geq 0$ for all $t \in[0,1]$.

Lemma 2.6 ([10, Lemma 2.7]) If $x(t) \in C[0,1]$ satisfies

$$
\left\{\begin{array}{l}
-D^{\alpha} x(t) \geq 0, \quad 0<t<1 \\
x(0)=0 \\
D^{\alpha-1} x(1) \geq 0
\end{array}\right.
$$

then $x(t) \geq 0$ for all $t \in[0,1]$.

Lemma 2.7 Let $M, N$ be nonnegative constants and $M \geq N$. If $u, v \in C[0,1]$ satisfy $D^{\beta} u(t), D^{\beta} v(t) \in C[0,1]$, and

$$
\left\{\begin{array}{l}
-D^{\beta} u(t) \geq-M u(t)+N v(t), \quad t \in[0, T), \\
-D^{\beta} v(t) \geq-M v(t)+N u(t), \quad t \in[0, T), \\
u(0)=0, \quad D^{\beta-1} u(1) \geq \lambda I^{\sigma} u(\eta), \\
v(0)=0, \quad D^{\beta-1} v(1) \geq \lambda I^{\sigma} v(\eta),
\end{array}\right.
$$

then $u(t) \geq 0$ and $v(t) \geq 0$ for all $t \in[0,1]$.

Proof Let $p(t)=u(t)+v(t), t \in[0,1]$. Then by (2.10) we have

$$
\left\{\begin{array}{l}
-D^{\beta} p(t) \geq-(M-N) p(t), \quad t \in[0,1] \\
p(0)=0 \\
D^{\beta-1} p(1) \geq \lambda I^{\sigma} p(\eta) .
\end{array}\right.
$$

Thus by (2.11) and Lemma 2.5 we have that

$$
p(t) \geq 0, \quad \forall t \in[0,1], \quad \text { i.e., } \quad u(t)+v(t) \geq 0, \quad \forall t \in[0,1] .
$$

Next, we show that $u(t) \geq 0$ and $v(t) \geq 0$ for all $t \in[0,1]$. Using (2.10) and (2.12), we find that

$$
\left\{\begin{array}{l}
-D^{\beta} u(t) \geq-(M+N) u(t), \quad t \in[0,1] \\
u(0)=0 \\
D^{\beta-1} u(1) \geq \lambda I^{\sigma} u(\eta)
\end{array}\right.
$$

which, in view of (2.13) and Lemma 2.5 , yield $u(t) \geq 0$ for all $t \in[0,1]$. In a similar way, we can show that $v(t) \geq 0$ for all $t \in[0,1]$. 


\section{Main results}

Theorem 3.1 Suppose that conditions $\left(H_{1}\right)-\left(H_{6}\right)$ hold. Then there is an extremal solution $\left(x^{*}, y^{*}\right) \in\left[x_{0}, y_{0}\right] \times\left[x_{0}, y_{0}\right]$ of the nonlinear problem (1.1). Moreover, there exist monotone iterative sequences $\left\{x_{n}\right\},\left\{y_{n}\right\} \subset\left[x_{0}, y_{0}\right]$ such that $x_{n} \rightarrow x^{*}, y_{n} \rightarrow y^{*}(n \rightarrow \infty)$ uniformly for $t \in[0,1]$ and

$$
x_{0} \leq x_{1} \leq \cdots \leq x_{n} \leq \cdots \leq x^{*} \leq y^{*} \leq \cdots \leq y_{n} \leq \cdots \leq y_{1} \leq y_{0} .
$$

Moreover, we have

$$
D^{\alpha} y_{0} \leq D^{\alpha} y_{1} \leq \cdots \leq D^{\alpha} y_{n} \leq \cdots \leq D^{\alpha} y^{*} \leq D^{\alpha} x^{*} \leq \cdots \leq D^{\alpha} x_{n} \leq \cdots \leq D^{\alpha} x_{1} \leq D^{\alpha} x_{0},
$$

where

$$
\left[x_{0}, y_{0}\right]=\left\{x \in C_{\alpha}[0,1]: x_{0}(t) \leq x(t) \leq y_{0}(t), t \in[0,1]\right\} .
$$

Proof For any $x_{n-1}, y_{n-1} \in C_{\alpha}[0,1], n \geq 1$, we define

$$
\left\{\begin{aligned}
\sigma_{n}^{1}(t)= & f\left(t, x_{n-1}(t), y_{n-1}(t), D^{\alpha} x_{n-1}(t), D^{\alpha} y_{n-1}(t)\right) \\
& +M \phi_{p}\left(-D^{\alpha} x_{n-1}(t)\right)+N \phi_{p}\left(-D^{\alpha} y_{n-1}(t)\right), \\
\sigma_{n}^{2}(t)= & g\left(t, y_{n-1}(t), x_{n-1}(t), D^{\alpha} y_{n-1}(t), D^{\alpha} x_{n-1}(t)\right) \\
& +M \phi_{p}\left(-D^{\alpha} y_{n-1}(t)\right)+N \phi_{p}\left(-D^{\alpha} x_{n-1}(t)\right) .
\end{aligned}\right.
$$

Consider (2.3) as follows:

$$
\left\{\begin{array}{l}
-D^{\beta}\left(\phi_{p}\left(-D^{\alpha} x_{n}(t)\right)\right)=\sigma_{n}^{1}(t)-M\left(\phi_{p}\left(-D^{\alpha} x_{n}(t)\right)\right)-N\left(\phi_{p}\left(-D^{\alpha} y_{n}(t)\right)\right), \\
-D^{\beta}\left(\phi_{p}\left(-D^{\alpha} y_{n}(t)\right)\right)=\sigma_{n}^{2}(t)-M\left(\phi_{p}\left(-D^{\alpha} y_{n}(t)\right)\right)-N\left(\phi_{p}\left(-D^{\alpha} x_{n}(t)\right)\right), \\
D^{\alpha} x_{n}(0)=0, \\
D^{\beta-1}\left(\phi_{p}\left(-D^{\alpha} x_{n}(1)\right)\right) \\
\quad=I^{\sigma}\left\{h\left(\eta, \phi_{p}\left(-D^{\alpha} x_{n-1}(\eta)\right)\right)+\lambda\left[\phi_{p}\left(-D^{\alpha} x_{n}(\eta)\right)-\phi_{p}\left(-D^{\alpha} x_{n-1}(\eta)\right)\right]\right\}+a_{1}, \\
x_{n}(0)=0, \quad D^{\alpha-1} x_{n}(1)=I^{\omega} x_{n-1}(\xi)+d_{1}, \\
D^{\alpha} y_{n}(0)=0, \\
D^{\beta-1}\left(\phi_{p}\left(-D^{\alpha} y_{n}(1)\right)\right) \\
\quad=I^{\sigma}\left\{k\left(\eta, \phi_{p}\left(-D^{\alpha} y_{n-1}(\eta)\right)\right)+\lambda\left[\phi_{p}\left(-D^{\alpha} y_{n}(\eta)\right)-\phi_{p}\left(-D^{\alpha} y_{n-1}(\eta)\right)\right]\right\}+a_{2}, \\
y_{n}(0)=0, \quad D^{\alpha-1} y_{n}(1)=I^{\omega} y_{n-1}(\xi)+d_{2} .
\end{array}\right.
$$

In view of Lemma 2.4, problem (3.1) has a unique solution in $C_{\alpha}[0,1] \times C_{\alpha}[0,1]$.

Now we show that $\left\{x_{n}(t)\right\}$ and $\left\{y_{n}(t)\right\}$ satisfy the relations

$$
x_{n-1} \leq x_{n} \leq y_{n} \leq y_{n-1} \quad \text { and } \quad D^{\alpha} y_{n-1} \leq D^{\alpha} y_{n} \leq D^{\alpha} x_{n} \leq D^{\alpha} x_{n-1}, \quad n=1,2, \ldots
$$


Let $u(t)=\phi_{p}\left(-D^{\alpha} x_{1}(t)\right)-\phi_{p}\left(-D^{\alpha} x_{0}(t)\right), v(t)=\phi_{p}\left(-D^{\alpha} y_{0}(t)\right)-\phi_{p}\left(-D^{\alpha} y_{1}(t)\right)$. By condition (3.1) and $\left(H_{1}\right)$ we have

$$
\begin{aligned}
-D^{\beta} u(t)= & -D^{\beta}\left(\phi_{p}\left(-D^{\alpha} x_{1}(t)\right)\right)+D^{\beta}\left(\phi_{p}\left(-D^{\alpha} x_{0}(t)\right)\right) \\
\geq & f\left(t, x_{0}(t), y_{0}(t), D^{\alpha} x_{0}(t), D^{\alpha} y_{0}(t)\right)+M \phi_{p}\left(-D^{\alpha} x_{0}(t)\right)+N \phi_{p}\left(-D^{\alpha} y_{0}(t)\right) \\
& -M \phi_{p}\left(-D^{\alpha} x_{1}(t)\right)-N \phi_{p}\left(D^{\alpha} y_{1}(t)\right)-f\left(t, x_{0}(t), y_{0}(t), D^{\alpha} x_{0}(t), D^{\alpha} y_{0}(t)\right) \\
= & -M u(t)+N v(t) .
\end{aligned}
$$

Also, $u(0)=0$, and

$$
\begin{aligned}
D^{\beta-1} u(1)= & D^{\beta-1}\left(\phi_{p}\left(-D^{\alpha} x_{1}(1)\right)\right)-D^{\beta-1}\left(\phi_{p}\left(-D^{\alpha} x_{0}(1)\right)\right) \\
\geq & I^{\sigma}\left\{h\left(\eta, \phi_{p}\left(-D^{\alpha} x_{0}(\eta)\right)\right)+\lambda\left[\phi_{p}\left(-D^{\alpha} x_{1}(\eta)\right)-\phi_{p}\left(-D^{\alpha} x_{0}(\eta)\right)\right]\right\}+a_{1} \\
& -I^{\sigma} h\left(\eta, \phi_{p}\left(-D^{\alpha} x_{0}(\eta)\right)\right)-a_{1} \\
= & \lambda I^{\sigma} u(\eta) .
\end{aligned}
$$

In a similar way, we can prove that

$$
-D^{\beta} v(t) \geq-M v(t)+N u(t), \quad v(0)=0, \quad D^{\beta-1} v(1) \geq \lambda I^{\sigma} v(\eta) .
$$

So, from the above inequality we have

$$
\left\{\begin{array}{l}
-D^{\beta} u(t) \geq-M u(t)+N v(t), \\
-D^{\beta} v(t) \geq-M v(t)+N u(t), \\
u(0)=0, \quad D^{\beta-1} u(1) \geq \lambda I^{\sigma} u(\eta), \\
v(0)=0, \quad D^{\beta-1} v(1) \geq \lambda I^{\sigma} v(\eta) .
\end{array}\right.
$$

Thus, in view of Lemma 2.7, we get $\phi_{p}\left(-D^{\alpha} x_{1}(t)\right) \geq \phi_{p}\left(-D^{\alpha} x_{0}(t)\right), \phi_{p}\left(-D^{\alpha} y_{0}(t)\right) \geq$ $\phi_{p}\left(-D^{\alpha} y_{1}(t)\right)$ for all $t \in[0,1]$. Since $\Phi_{p}(x)$ is nondecreasing, we have $D^{\alpha} x_{1}(t) \leq D^{\alpha} x_{0}(t)$ and $D^{\alpha} y_{0}(t) \leq D^{\alpha} y_{1}(t)$ for all $t \in[0,1]$.

Let $\epsilon(t)=x_{1}(t)-x_{0}(t), \theta(t)=y_{0}(t)-y_{1}(t)$. From (3.1) and $\left(H_{1}\right)$ we have

$$
\left\{\begin{array}{l}
-D^{\alpha} \epsilon(t) \geq 0, \quad t \in(0,1], \\
\epsilon(0)=0, \\
D^{\alpha-1} \epsilon(1) \geq I^{\omega} x_{0}(\xi)+d_{1}-I^{\omega} x_{0}(\xi)-d_{1}=0,
\end{array}\right.
$$

and

$$
\left\{\begin{array}{l}
-D^{\alpha} \theta(t) \geq 0, \quad t \in(0,1] \\
\theta(0)=0, \\
D^{\alpha-1} \theta(1) \geq 0 .
\end{array}\right.
$$

By Lemma 2.6 we have $x_{1}(t) \geq x_{0}(t)$ and $y_{0}(t) \geq y_{1}(t)$ for all $t \in[0,1]$. 
Now we put $w(t)=\phi_{p}\left(-D^{\alpha} y_{1}(t)\right)-\phi_{p}\left(-D^{\alpha} x_{1}(t)\right)$. Applying $\left(H_{2}\right),\left(H_{3}\right)$, and (3.1), we obtain

$$
\begin{aligned}
-D^{\beta} w(t)= & g\left(t, y_{0}(t), x_{0}(t), D^{\alpha} y_{0}(t), D^{\alpha} x_{0}(t)\right)+M \phi_{p}\left(-D^{\alpha} y_{0}(t)\right)+N \phi_{p}\left(-D^{\alpha} x_{0}(t)\right) \\
& -M \phi_{p}\left(-D^{\alpha} y_{1}(t)\right)-N \phi_{p}\left(-D^{\alpha} x_{1}(t)\right)-f\left(t, x_{0}(t), y_{0}(t), D^{\alpha} x_{0}(t), D^{\alpha} y_{0}(t)\right) \\
& -M \phi_{p}\left(-D^{\alpha} x_{0}(t)\right)-N \phi_{p}\left(-D^{\alpha} y_{0}(t)\right)+M \phi_{p}\left(-D^{\alpha} x_{1}(t)\right)+N \phi_{p}\left(-D^{\alpha} y_{1}(t)\right) \\
\geq & -M\left[\phi_{p}\left(-D^{\alpha} y_{0}(t)\right)-\phi_{p}\left(-D^{\alpha} x_{0}(t)\right)\right]-N\left[\phi_{p}\left(-D^{\alpha} x_{0}(t)\right)-\phi_{p}\left(-D^{\alpha} y_{0}(t)\right)\right] \\
& +M \phi_{p}\left(-D^{\alpha} y_{0}(t)\right)+N \phi_{p}\left(-D^{\alpha} x_{0}(t)\right)-M \phi_{p}\left(-D^{\alpha} y_{1}(t)\right)-N \phi_{p}\left(-D^{\alpha} x_{1}(t)\right) \\
& -M \phi_{p}\left(-D^{\alpha} x_{0}(t)\right)-N \phi_{p}\left(-D^{\alpha} y_{0}(t)\right)+M \phi_{p}\left(-D^{\alpha} x_{1}(t)\right)+N \phi_{p}\left(-D^{\alpha} y_{1}(t)\right) \\
= & -(M-N) w(t) .
\end{aligned}
$$

Also, $w(0)=\phi_{p}\left(-D^{\alpha} y_{1}(0)\right)-\phi_{p}\left(-D^{\alpha} x_{1}(0)\right)=0$, and

$$
\begin{aligned}
D^{\beta-1} w(1)= & I^{\sigma}\left\{k\left(\eta, \phi_{p}\left(-D^{\alpha} y_{0}(\eta)\right)\right)+\lambda\left[\phi_{p}\left(-D^{\alpha} y_{1}(\eta)\right)-\phi_{p}\left(-D^{\alpha} y_{0}(\eta)\right)\right]\right\}+a_{2} \\
& -I^{\sigma}\left\{h\left(\eta, \phi_{p}\left(-D^{\alpha} x_{0}(\eta)\right)\right)+\lambda\left[\phi_{p}\left(-D^{\alpha} x_{1}(\eta)\right)-\phi_{p}\left(-D^{\alpha} x_{0}(\eta)\right)\right]\right\}-a_{1} \\
\geq & I^{\sigma}\left\{\lambda\left[\phi_{p}\left(-D^{\alpha} y_{0}(\eta)\right)-\phi_{p}\left(-D^{\alpha} x_{0}(\eta)\right)\right]+\lambda\left[\phi_{p}\left(-D^{\alpha} y_{1}(\eta)\right)-\phi_{p}\left(-D^{\alpha} y_{0}(\eta)\right)\right]\right. \\
& \left.-\lambda\left[\phi_{p}\left(-D^{\alpha} x_{1}(\eta)\right)-\phi_{p}\left(-D^{\alpha} x_{0}(\eta)\right)\right]\right\}+\left(a_{2}-a_{1}\right) \\
\geq & \lambda I^{\sigma} w(\eta) .
\end{aligned}
$$

In view of Lemma 2.5, we have that $w(t) \geq 0$ for all $t \in[0,1]$. Thus we have the relation $\phi_{p}\left(-D^{\alpha} x_{1}(t)\right) \leq \phi_{p}\left(-D^{\alpha} y_{1}(t)\right)$, that is, $D^{\alpha} x_{1}(t) \geq D^{\alpha} y_{1}(t)$, since $\Phi_{p}(x)$ is nondecreasing. Therefore $D^{\alpha} y_{0}(t) \leq D^{\alpha} y_{1}(t) \leq D^{\alpha} x_{1}(t) \leq D^{\alpha} x_{0}(t)$ for all $t \in[0,1]$.

Let $\delta(t)=y_{1}(t)-x_{1}(t)$. It follows from (3.1) that

$$
\left\{\begin{array}{l}
-D^{\alpha} \delta(t)=-D^{\alpha} y_{1}(t)+D^{\alpha} x_{1}(t) \geq 0 \\
\delta(0)=0 \\
D^{\alpha-1} \delta(1)=I^{\omega} y_{0}(\xi)+d_{2}-I^{\omega} x_{0}(\xi)-d_{1} \geq 0
\end{array}\right.
$$

By Lemma 2.6 we obtain $y_{1}(t) \geq x_{1}(t)$ for all $t \in[0,1]$. Hence we have the relation $x_{0}(t) \leq$ $x_{1}(t) \leq y_{1}(t) \leq y_{0}(t)$.

Now we assume that

$$
x_{k-1} \leq x_{k} \leq y_{k} \leq y_{k-1} \quad \text { and } \quad D^{\alpha} y_{k-1} \leq D^{\alpha} y_{k} \leq D^{\alpha} x_{k} \leq D^{\alpha} x_{k-1} \quad \text { for some } k \geq 1
$$

We will prove that (3.2) is also true for $k+1$. Let

$$
\begin{aligned}
& u(t)=\phi_{p}\left(-D^{\alpha} x_{k+1}(t)\right)-\phi_{p}\left(-D^{\alpha} x_{k}(t)\right), \quad v(t)=\phi_{p}\left(-D^{\alpha} y_{k}(t)\right)-\phi_{p}\left(-D^{\alpha} y_{k+1}(t)\right), \\
& w(t)=\phi_{p}\left(-D^{\alpha} y_{k+1}(t)\right)-\phi_{p}\left(-D^{\alpha} x_{k+1}(t)\right), \quad \epsilon(t)=x_{k+1}(t)-x_{k}(t), \\
& \theta(t)=y_{k}(t)-y_{k+1}(t), \quad \delta(t)=y_{k+1}(t)-x_{k+1}(t) .
\end{aligned}
$$


By $\left(H_{2}\right),\left(H_{3}\right)$, and (3.1) we have that

$$
\begin{aligned}
& \left\{\begin{array}{l}
-D^{\beta} u(t) \geq-M u(t)+N v(t) \\
-D^{\beta} v(t) \geq-M v(t)+N u(t) \\
u(0)=0, \quad D^{\beta-1} u(1) \geq \lambda I^{\sigma} u(\eta) \\
v(0)=0, \quad D^{\beta-1} v(1) \geq \lambda I^{\sigma} v(\eta)
\end{array}\right. \\
& \left\{\begin{array}{l}
-D^{\alpha} \epsilon(t) \geq 0 \\
\epsilon(0)=0 \\
D^{\alpha-1} \epsilon(1) \geq 0
\end{array}\right. \\
& \left\{\begin{array}{l}
-D^{\alpha} \theta(t) \geq 0 \\
\theta(0)=0 \\
D^{\alpha-1} \theta(1) \geq 0
\end{array}\right.
\end{aligned}
$$

and

$$
\begin{aligned}
& \left\{\begin{array}{l}
-D^{\beta} w(t) \geq-(M-N) w(t) \\
w(0)=0 \\
D^{\beta-1} w(1) \geq \lambda I^{\sigma} w(\eta)
\end{array}\right. \\
& \left\{\begin{array}{l}
-D^{\alpha} \delta(t) \geq 0 \\
\delta(0)=0 \\
D^{\alpha-1} \delta(1) \geq 0
\end{array}\right.
\end{aligned}
$$

In view of Lemmas 2.5-2.7, we obtain

$$
x_{k} \leq x_{k+1} \leq y_{k+1} \leq y_{k} \quad \text { and } \quad D^{\alpha} y_{k} \leq D^{\alpha} y_{k+1} \leq D^{\alpha} x_{k+1} \leq D^{\alpha} x_{k}, \quad \forall t \in[0,1]
$$

From the above, by induction, it is not difficult to prove that $x_{0} \leq x_{1} \leq \cdots \leq x_{n} \leq \cdots \leq$ $y_{n} \leq \cdots \leq y_{1} \leq y_{0}$ and $D^{\alpha} y_{0} \leq D^{\alpha} y_{1} \leq \cdots \leq D^{\alpha} y_{n} \leq \cdots \leq D^{\alpha} x_{n} \leq \cdots \leq D^{\alpha} x_{1} \leq D^{\alpha} x_{0}$.

Since the solution space is $C_{\alpha}[0,1]$, the sequences $\left\{x_{n}\right\}$ and $\left\{y_{n}\right\}$ are uniformly bounded and equicontinuous. The Arzelà-Ascoli theorem guarantees that they are relatively compact sets in the space $C_{\alpha}[0,1]$. Therefore $\left\{x_{n}\right\}$ and $\left\{y_{n}\right\}$ converge to $x^{*}(t)$ and $y^{*}(t)$ uniformly on $[0,1]$, respectively, that is,

$$
\lim _{n \rightarrow \infty} x_{n}(t)=x^{*}(t), \quad \lim _{n \rightarrow \infty} y_{n}(t)=y^{*}(t), \quad \forall t \in[0,1]
$$

and

$$
\lim _{n \rightarrow \infty} D^{\alpha} x_{n}(t)=D^{\alpha} x^{*}(t), \quad \lim _{n \rightarrow \infty} D^{\alpha} y_{n}(t)=D^{\alpha} y^{*}(t), \quad \forall t \in[0,1]
$$

uniformly in $t \in[0,1]$. Moreover, from (3.1) and (3.2) we obtain that $x^{*}(t)$ and $y^{*}(t)$ are solutions of problem (1.1). 
Finally, we show that $\left(x^{*}, y^{*}\right)$ is an extremal solution of system (1.1). Let $(x, y) \in\left[x_{0}, y_{0}\right] \times$ $\left[x_{0}, y_{0}\right]$ be any solution of problem (1.1), that is,

$$
\left\{\begin{aligned}
-D^{\beta}\left(\phi_{p}\left(-D^{\alpha} x(t)\right)\right) & =f\left(t, x(t), y(t), D^{\alpha} x(t), D^{\alpha} y(t)\right), \quad t \in(0,1], \\
-D^{\beta}\left(\phi_{p}\left(-D^{\alpha} y(t)\right)\right) & \left.=g\left(t, y(t), x(t), D^{\alpha} y(t)\right), D^{\alpha} x(t)\right), \quad t \in(0,1], \\
D^{\alpha} x(0)=0, & \begin{array}{rl}
D^{\beta-1}\left(\phi_{p}\left(-D^{\alpha} x(1)\right)\right) & =I^{\sigma} h\left(\eta, \phi_{p}\left(-D^{\alpha} x(\eta)\right)\right)+a_{1} \\
& =\frac{1}{\Gamma(\sigma)} \int_{0}^{\eta}(\eta-s)^{\sigma-1} h\left(s, \phi_{p}\left(-D^{\alpha} x(s)\right)\right) d s+a_{1}, \\
x(0)=0, \quad D^{\alpha-1} x(1)=I^{\omega} x(\xi)+d_{1}=\frac{1}{\Gamma(\omega)} \int_{0}^{\xi}(\xi-s)^{\omega-1} x(s) d s+d_{1}, & \\
D^{\alpha} y(0)=0, \quad & =\frac{1}{\Gamma(\sigma)} \int_{0}^{\eta}(\eta-s)^{\sigma-1} k\left(s, \phi_{p}\left(-D^{\alpha} y(s)\right)\right) d s+a_{2}, \\
D^{\beta-1}\left(\phi_{p}\left(-D^{\alpha} y(1)\right)\right) & =I^{\sigma} k\left(\eta, \phi_{p}\left(-D^{\alpha} y(\eta)\right)\right)+a_{2} \\
y(0)=0, \quad D^{\alpha-1} y(1)=I^{\omega} y(\xi)+d_{2}=\frac{1}{\Gamma(\omega)} \int_{0}^{\xi}(\xi-s)^{\omega-1} y(s) d s+d_{2} .
\end{array}
\end{aligned}\right.
$$

Applying (3.1), (3.5), $\left(H_{2}\right),\left(H_{3}\right)$, Lemma 2.6, and Lemma 2.7, we have

$$
x_{n} \leq x, \quad y \leq y_{n}, \quad D^{\alpha} x \leq D^{\alpha} x_{n}, \quad D^{\alpha} y_{n} \leq D^{\alpha} y, \quad n=1,2, \ldots
$$

Taking the limit as $n \rightarrow \infty$ in (3.6), we have $x^{*} \leq x, y \leq y^{*}$, that is, $\left(x^{*}, y^{*}\right)$ is an extremal solution of system $(1.1)$ in $\left[x_{0}, y_{0}\right] \times\left[x_{0}, y_{0}\right]$. This completes the proof.

\section{Iteration procedure and a numerical example}

In this section, we introduce a numerical procedure to obtain an appropriate solution of (1.1). Define

$$
E(n)=\left\|x_{n}(t)-y_{n}(t)\right\|_{1}=\int_{0}^{1}\left|x_{n}(t)-y_{n}(t)\right| d t .
$$

For the iteration Eq. (3.1), let $\phi_{p}\left(-D^{\alpha} x_{n}(t)\right)=u_{n}$. Then $-D^{\alpha} x_{n}(t)=\phi_{q}\left(u_{n}\right)$, and with the boundary conditions $x_{n}(0)=0$ and $D^{\alpha-1} x_{n}(1)=D^{\frac{2}{3}} x_{n}(1)=I^{\omega} x_{n-1}(\xi)+d_{1}=l_{1}$, by Lemma 2.3 we have

$$
x_{n}(t)=\frac{l_{1}}{\Gamma(\alpha)} t^{\alpha-1}+\int_{0}^{1} H(t, s) \phi_{q}\left(u_{n}(s)\right) d s,
$$

where $l_{1}=\frac{1}{\Gamma(\omega)} \int_{0}^{\xi}(\xi-s)^{\omega-1} x_{n-1}(s) d s+d_{1}$ and

$$
H(t, s)= \begin{cases}t^{\alpha-1}-(t-s)^{\alpha-1}, & 0 \leq s \leq t \leq 1 \\ t^{\alpha-1}, & 0 \leq t \leq s \leq 1\end{cases}
$$

We can also put $\phi_{p}\left(-D^{\alpha} y_{n}(t)\right)=v_{n}$. Then $-D^{\alpha} y_{n}(t)=\phi_{q}\left(v_{n}\right)$. In a similar way, we can prove that

$$
y_{n}(t)=\frac{l_{2}}{\Gamma(\alpha)} t^{\alpha-1}+\int_{0}^{1} H(t, s) \phi_{q}\left(v_{n}(s)\right) d s,
$$


where $l_{2}=\frac{1}{\Gamma(\omega)} \int_{0}^{\xi}(\xi-s)^{\omega-1} y_{n-1}(s) d s+d_{2}=1.1284 \int_{0}^{\frac{1}{2}}\left(\frac{1}{2}-s\right)^{\frac{1}{2}} y_{n-1}(s) d s+0.004$. Thus the iteration Eq. (3.1) can be rewritten as

$$
\left\{\begin{array}{l}
-D^{\beta} u_{n}=-M u_{n}-N v_{n}+f\left(t, x_{n-1}, y_{n-1},-\phi_{q}\left(u_{n-1}\right),-\phi_{q}\left(v_{n-1}\right)\right)+M u_{n-1}+N v_{n-1}, \\
-D^{\beta} v_{n}=-M v_{n}-N u_{n}+g\left(t, y_{n-1}, x_{n-1},-\phi_{q}\left(v_{n-1}\right),-\phi_{q}\left(u_{n-1}\right)\right)+M v_{n-1}+N u_{n-1}, \\
u_{n}(0)=0, \quad D^{\beta-1} u_{n}(1)=\lambda I^{\sigma} u_{n}(\eta)+b_{1}, \\
v_{n}(0)=0, \quad D^{\beta-1} v_{n}(1)=\lambda I^{\sigma} v_{n}(\eta)+b_{2} .
\end{array}\right.
$$

Applying Lemma 2.1 to (4.3), we obtain

$$
\left\{\begin{aligned}
u_{n}(t)= & \frac{b_{1} \Gamma(\beta+\sigma)}{\Gamma(\beta)\left[\Gamma(\beta+\sigma)-\lambda \eta^{\beta+\sigma-1}\right]} t^{\beta-1} \\
& +\int_{0}^{1} G(t, s)\left[-M u_{n}(s)-N v_{n}(s)+f\left(s, x_{n-1}(s), y_{n-1}(s),-\phi_{q}\left(u_{n-1}(s)\right),\right.\right. \\
& \left.\left.-\phi_{q}\left(v_{n-1}(s)\right)\right)+M u_{n-1}(s)+N v_{n-1}(s)\right] d s \\
v_{n}(t)= & \frac{b_{2} \Gamma(\beta+\sigma)}{\Gamma(\beta)\left[\Gamma(\beta+\sigma)-\lambda \eta^{\beta+\sigma-1}\right]} t^{\beta-1} \\
& +\int_{0}^{1} G(t, s)\left[-M v_{n}(s)-N u_{n}(s)+g\left(s, y_{n-1}(s), x_{n-1}(s),-\phi_{q}\left(v_{n-1}(s)\right),\right.\right. \\
& \left.\left.-\phi_{q}\left(u_{n-1}(s)\right)\right)+M v_{n-1}(s)+N u_{n-1}(s)\right] d s
\end{aligned}\right.
$$

where $b_{1}=I^{\sigma} h\left(\eta, u_{n-1}(\eta)\right)-\lambda I^{\sigma} u_{n-1}(\eta)+a_{1}, b_{2}=I^{\sigma} k\left(\eta, v_{n-1}(\eta)\right)-\lambda I^{\sigma} v_{n-1}(\eta)+a_{2}$, and

$$
G(t, s)=\frac{1}{\Delta} \begin{cases}{\left[\Gamma(\beta+\sigma)-\lambda(\eta-s)^{\beta+\sigma-1}\right] t^{\beta-1}} & \\ \quad-\left[\Gamma(\beta+\sigma)-\lambda \eta^{\beta+\sigma-1}\right](t-s)^{\beta-1}, & s \leq t, s \leq \eta, \\ \Gamma(\beta+\sigma) t^{\beta-1}-\lambda(\eta-s)^{\beta+\sigma-1} t^{\beta-1}, & t \leq s \leq \eta, \\ \Gamma(\beta+\sigma)\left[t^{\beta-1}-(t-s)^{\beta-1}\right]+\lambda \eta^{\beta+\sigma-1}(t-s)^{\beta-1}, & \eta \leq s \leq t, \\ \Gamma(\beta+\sigma) t^{\beta-1}, & s \geq t, s \geq \eta,\end{cases}
$$

$\Delta=\Gamma(\beta)\left[\Gamma(\beta+\sigma)-\lambda \eta^{\beta+\sigma-1}\right]$.

Discretize the interval $[0,1]$ with the nodes $t_{i}=i h, h=\frac{1}{K}, K \in \mathbb{N}$. Let $x_{n}^{(i)} \approx x_{n}\left(t_{i}\right), u_{n}^{(i)} \approx$ $u_{n}\left(t_{i}\right), H(i, j)=H\left(t_{i}, s_{j}\right), G(i, j)=G\left(t_{i}, s_{j}\right)$, and

$$
\left\{\begin{array}{l}
f_{n-1}^{(j)}=f\left(s_{j}, x_{n-1}\left(s_{j}\right), y_{n-1}\left(s_{j}\right),-\phi_{q}\left(u_{n-1}\left(s_{j}\right)\right),-\phi_{q}\left(v_{n-1}\left(s_{j}\right)\right)\right)+M u_{n-1}\left(s_{j}\right)+N v_{n-1}\left(s_{j}\right), \\
g_{n-1}^{(j)}=g\left(s_{j}, y_{n-1}\left(s_{j}\right), x_{n-1}\left(s_{j}\right),-\phi_{q}\left(v_{n-1}\left(s_{j}\right)\right),-\phi_{q}\left(u_{n-1}\left(s_{j}\right)\right)\right)+M v_{n-1}\left(s_{j}\right)+N u_{n-1}\left(s_{j}\right) .
\end{array}\right.
$$

Using the trapezoidal quadrature rule to approximate the integrals in the right-hand sides of (4.4), (4.2), and (4.1), we obtain the following linear systems of equations:

$$
\left\{\begin{aligned}
u_{n}^{(i)}= & \frac{b_{1} \Gamma(\beta+\sigma)}{\Gamma(\beta)\left[\Gamma(\beta+\sigma)-\lambda \eta^{\beta+\sigma-1}\right]} t_{i}^{\beta-1}-\frac{h}{2} \sum_{j=0}^{K} M G(i, j) d_{j} u_{n}^{(j)} \\
& -\frac{h}{2} \sum_{j=0}^{K} N G(i, j) d_{j} v_{n}^{(j)}+\frac{h}{2} \sum_{j=0}^{K} G(i, j) d_{j} f_{n-1}^{(j)} \\
v_{n}^{(i)}= & \frac{b_{2} \Gamma(\beta+\sigma)}{\Gamma(\beta)\left[\Gamma(\beta+\sigma)-\lambda \eta^{\beta+\sigma-1}\right]} t_{i}^{\beta-1}-\frac{h}{2} \sum_{j=0}^{K} M G(i, j) d_{j} v_{n}^{(j)} \\
& -\frac{h}{2} \sum_{j=0}^{K} N G(i, j) d_{j} u_{n}^{(j)}+\frac{h}{2} \sum_{j=0}^{K} G(i, j) d_{j} g_{n-1}^{(j)}
\end{aligned}\right.
$$

and

$$
\left\{\begin{array}{l}
x_{n}^{(i)}=\frac{l_{1}}{\Gamma(\alpha)} t_{i}^{\alpha-1}+\frac{h}{2} \sum_{j=0}^{K} H(i, j) d_{j} \phi_{q}\left(u_{n}^{(j)}\right) \\
y_{n}^{(i)}=\frac{l_{2}}{\Gamma(\alpha)} t_{i}^{\alpha-1}+\frac{h}{2} \sum_{j=0}^{K} H(i, j) d_{j} \phi_{q}\left(v_{n}^{(j)}\right)
\end{array}\right.
$$


for the unknown $u_{n}^{(i)}, x_{n}^{(i)}, 0 \leq i \leq K$, where $\left\{d_{j}\right\}$ are the coefficients in the rule, $d_{0}=d_{K}=1$, and $d_{j}=2$ for $1 \leq j \leq K-1$.

Setting $G_{i j}=\frac{h}{2} \sum_{j=0}^{K} G(i, j) d_{j}, H_{i j}=\frac{h}{2} \sum_{j=0}^{K} H(i, j) d_{j}$, the matrix $\Phi=\left(G_{i j}\right)$, and $B=\left(H_{i j}\right)$ with the identity matrix $\mathbb{I}$. Systems (4.5) and (4.6) can be written as a system of matrixvector equations

$$
\left\{\begin{array}{l}
(I+M \Phi) \vec{U}_{n}+N \Phi \vec{v}_{n}=\frac{b_{1} \Gamma(\beta+\sigma)}{\Gamma(\beta)\left[\Gamma(\beta+\sigma)-\lambda \eta^{\beta+\sigma-1}\right]} S^{\beta-1}+\vec{F}_{n-1}, \\
(I+M \Phi) \vec{V}_{n}+N \Phi \vec{u}_{n}=\frac{b_{2} \Gamma(\beta+\sigma)}{\Gamma(\beta)\left[\Gamma(\beta+\sigma)-\lambda \eta^{\beta+\sigma-1}\right]} S^{\beta-1}+\vec{G}_{n-1} \\
\vec{X}_{n}=\frac{l_{1}}{\Gamma(\alpha)} S^{\alpha-1}+B \phi_{q}\left(\vec{U}_{n}\right), \\
\vec{Y}_{n}=\frac{l_{2}}{\Gamma(\alpha)} S^{\alpha-1}+B \phi_{q}\left(\vec{V}_{n}\right),
\end{array}\right.
$$

where $\vec{X}_{n}=\left[x_{n}^{(0)}, x_{n}^{(1)}, \ldots, x_{n}^{(K)}\right], \vec{Y}_{n}=\left[y_{n}^{(0)}, y_{n}^{(1)}, \ldots, y_{n}^{(K)}\right], \vec{U}_{n}=\left[u_{n}^{(0)}, u_{n}^{(1)}, \ldots, u_{n}^{(K)}\right], \vec{V}_{n}=$ $\left[v_{n}^{(0)}, v_{n}^{(1)}, \ldots, v_{n}^{(K)}\right], S=\left[t_{0}, t_{1}, \ldots, t_{K}\right]^{T}$, and $\vec{F}_{n-1}, \vec{G}_{n-1}$ are column vectors of their components $F_{n-1}^{(i)}=\frac{h}{2} \sum_{j=0}^{K} G(i, j) d_{j} f_{n-1}^{(j)}, G_{n-1}^{(i)}=\frac{h}{2} \sum_{j=0}^{K} G(i, j) d_{j} g_{n-1}^{(j)}$.

Example 4.1 Consider the following problem:

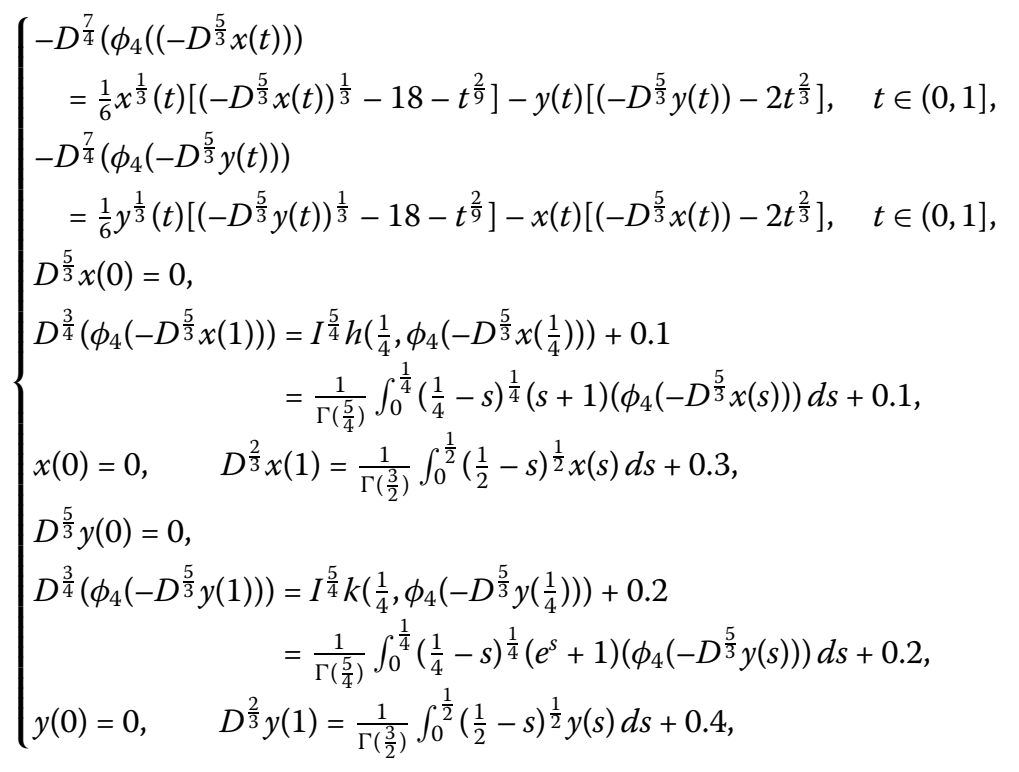

where $\beta=\frac{7}{4}, \alpha=\frac{5}{3}, \sigma=\frac{5}{4}, \omega=\frac{3}{2}, \eta=\frac{1}{4}, \xi=\frac{1}{2}, a_{1}=0.1, a_{2}=0.2, d_{1}=0.003, d_{2}=0.004$, $p=4$, and

$$
\left\{\begin{array}{l}
f\left(t, x(t), y(t), D^{\frac{5}{3}} x(t), D^{\frac{5}{3}} y(t)\right) \\
\quad=\frac{1}{6} x^{\frac{1}{3}}(t)\left[\left(-D^{\frac{5}{3}} x(t)\right)^{\frac{1}{3}}-18-t^{\frac{2}{9}}\right]-y(t)\left[\left(-D^{\frac{5}{3}} y(t)\right)-2 t^{\frac{2}{3}}\right] \\
g\left(t, y(t), x(t), D^{\frac{5}{3}} y(t), D^{\frac{5}{3}} x(t)\right) \\
\quad=\frac{1}{6} y^{\frac{1}{3}}(t)\left[\left(-D^{\frac{5}{3}} y(t)\right)^{\frac{1}{3}}-18-t^{\frac{2}{9}}\right]-x(t)\left[\left(-D^{\frac{5}{3}} x(t)\right)-2 t^{\frac{2}{3}}\right] \\
h\left(t, \phi_{4}\left(-D^{\frac{5}{3}} x\right)\right)=(t+1)\left(\phi_{4}\left(-D^{\frac{5}{3}} x\right)\right), \\
k\left(t, \phi_{4}\left(-D^{\frac{5}{3}} y\right)\right)=\left(e^{t}+1\right)\left(\phi_{4}\left(-D^{\frac{5}{3}} y\right)\right) .
\end{array}\right.
$$


Take $x_{0}(t)=0$ and $y_{0}(t)=3 t^{\frac{2}{3}}-\frac{9 \Gamma\left(\frac{2}{3}\right)}{14 \Gamma\left(\frac{1}{3}\right)} t^{\frac{7}{3}}$. Then $-1 \leq-t^{\frac{2}{3}}=D^{\frac{5}{3}} y_{0}(t) \leq D^{\frac{5}{3}} x_{0}(t)=0$. It is not difficult to verify that $\left(H_{1}\right)$ holds.

Since the function $\sqrt[3]{x}+x^{3}$ is increasing for $x \in R$, we obtain

$$
\begin{aligned}
f( & \left.t, \overline{x(t)}, \overline{y(t)}, D^{\frac{5}{3}} \overline{x(t)}, D^{\frac{5}{3}} \overline{y(t)}\right)-f\left(t, x(t), y(t), D^{\frac{5}{3}} x(t), D^{\frac{5}{3}} y(t)\right) \\
= & \frac{1}{6} \overline{x(t)^{\frac{1}{3}}}\left[\left(-D^{\frac{5}{3}} \overline{x(t)}\right)^{\frac{1}{3}}-600 t^{\frac{1}{100}}-t^{\frac{1}{9}}\right]-\overline{y(t)}\left[\left(-D^{\frac{5}{3}} \overline{y(t)}\right)-2 t^{\frac{1}{3}}\right] \\
& -\frac{1}{6} x^{\frac{1}{3}}(t)\left[\left(-D^{\frac{5}{3}} x(t)\right)^{\frac{1}{3}}-600 t^{\frac{1}{100}}-t^{\frac{1}{9}}\right]+y(t)\left[\left(-D^{\frac{5}{3}} y(t)\right)-2 t^{\frac{1}{3}}\right], \\
\leq & \frac{1}{6} x^{\frac{1}{3}}(t)\left[\left(-D^{\frac{5}{3}} \overline{x(t)}\right)^{\frac{1}{3}}-\left(-D^{\frac{5}{3}} x(t)\right)^{\frac{1}{3}}\right] \\
\leq & \frac{1}{6} \sqrt[3]{3}\left[\left(-D^{\frac{5}{3}} x(t)\right)^{3}-\left(-D^{\frac{5}{3}} \overline{x(t)}\right)^{3}\right] \\
= & \frac{1}{6} \sqrt[3]{3}\left[\Phi_{4}\left(-D^{\frac{5}{3}} x(t)\right)-\Phi_{4}\left(-D^{\frac{5}{3}} \overline{x(t)}\right)\right], \\
k(t, & \left.\phi_{4}\left(-D^{\frac{5}{3}} y\right)\right)-k\left(t, \phi_{4}\left(-D^{\frac{5}{3}} x\right)\right) \\
= & \left(e^{t}+1\right)\left(\phi_{4}\left(-D^{\frac{5}{3}} y\right)\right)-\left(e^{t}+1\right)\left(\phi_{4}\left(-D^{\frac{5}{3}} x\right)\right) \\
= & \left(e^{t}+1\right)\left[\phi_{4}\left(-D^{\frac{5}{3}} y\right)-\left(\phi_{4}\left(-D^{\frac{5}{3}} x\right)\right)\right] \\
\geq & (t+1)\left[\phi_{4}\left(-D^{\frac{5}{3}} y\right)-\left(\phi_{4}\left(-D^{\frac{5}{3}} x\right)\right)\right] \\
\geq & \phi_{4}\left(-D^{\frac{5}{3}} y\right)-\left(\phi_{4}\left(-D^{\frac{5}{3}} x\right)\right),
\end{aligned}
$$

where $x_{0}(t) \leq \overline{x(t)} \leq x(t) \leq y_{0}(t), x_{0}(t) \leq y(t) \leq \overline{y(t)} \leq y_{0}(t)$, and $x_{0}(t) \leq x(t) \leq y(t) \leq y_{0}(t)$. Thus $\left(H_{2}\right)$ and $\left(H_{3}\right)$ hold. From (4.9) and (4.10) we have $M=\frac{1}{6} \sqrt[3]{3}, N=0$, and $\lambda=1$. Then

$$
\begin{aligned}
& \Gamma(\beta+\sigma)=\Gamma\left(\frac{7}{4}+\frac{5}{4}\right)=\Gamma(3)=2>\lambda \eta^{\beta+\sigma-1}=1 \cdot\left(\frac{1}{4}\right)^{2}=0.0625 \\
& 2 \Gamma(\beta+\sigma)(M+N) \\
& \quad=2 \cdot \Gamma(3) \cdot \frac{\sqrt[3]{3}}{6} \approx 0.9614<\Gamma(\beta)\left[\Gamma(\beta+\sigma)-\lambda \eta^{\beta+\sigma-1}\right] \\
& \quad=\Gamma\left(\frac{7}{4}\right)\left[\Gamma(3)-1 \cdot\left(\frac{1}{4}\right)^{2}\right] \approx 1.7808, \\
& \Gamma(2-\beta) \lambda \eta^{\sigma}=\Gamma\left(\frac{1}{4}\right) \cdot 1 \cdot\left(\frac{1}{4}\right)^{\frac{5}{4}} \approx 0.6410<\Gamma(\sigma)=\Gamma\left(\frac{5}{4}\right) \approx 0.9064,
\end{aligned}
$$

which show that $\left(H_{4}\right),\left(H_{5}\right)$, and $\left(H_{6}\right)$ hold. Thus all conditions of Theorem 3.1 are satisfied. In consequence, the nonlinear system (4.8) has an extremal solution $\left(x^{*}, y^{*}\right) \in$ $\left[x_{0}(t), y_{0}(t)\right] \times\left[x_{0}(t), y_{0}(t)\right]$. Moreover, for this example, we found that for $\delta=10^{-10}$, which took $N=16$ iterations for $E(N)<\delta$. The graphs of $x_{n}$ and $y_{n}$ for some values of $n$ are shown in Table 1 and Fig. 1. 
Table $1 E(n)=3 n+1, n=0,1,2,3,4,5$

\begin{tabular}{lllllll}
\hline$n$ & 1 & 4 & 7 & 10 & 13 & 16 \\
\hline$E(n)$ & 0.6696 & 0.0096 & $2.6171 \mathrm{e}-04$ & $2.3360 \mathrm{e}-07$ & $1.5608 \mathrm{e}-8$ & $1.3942 \mathrm{e}-11$ \\
\hline
\end{tabular}

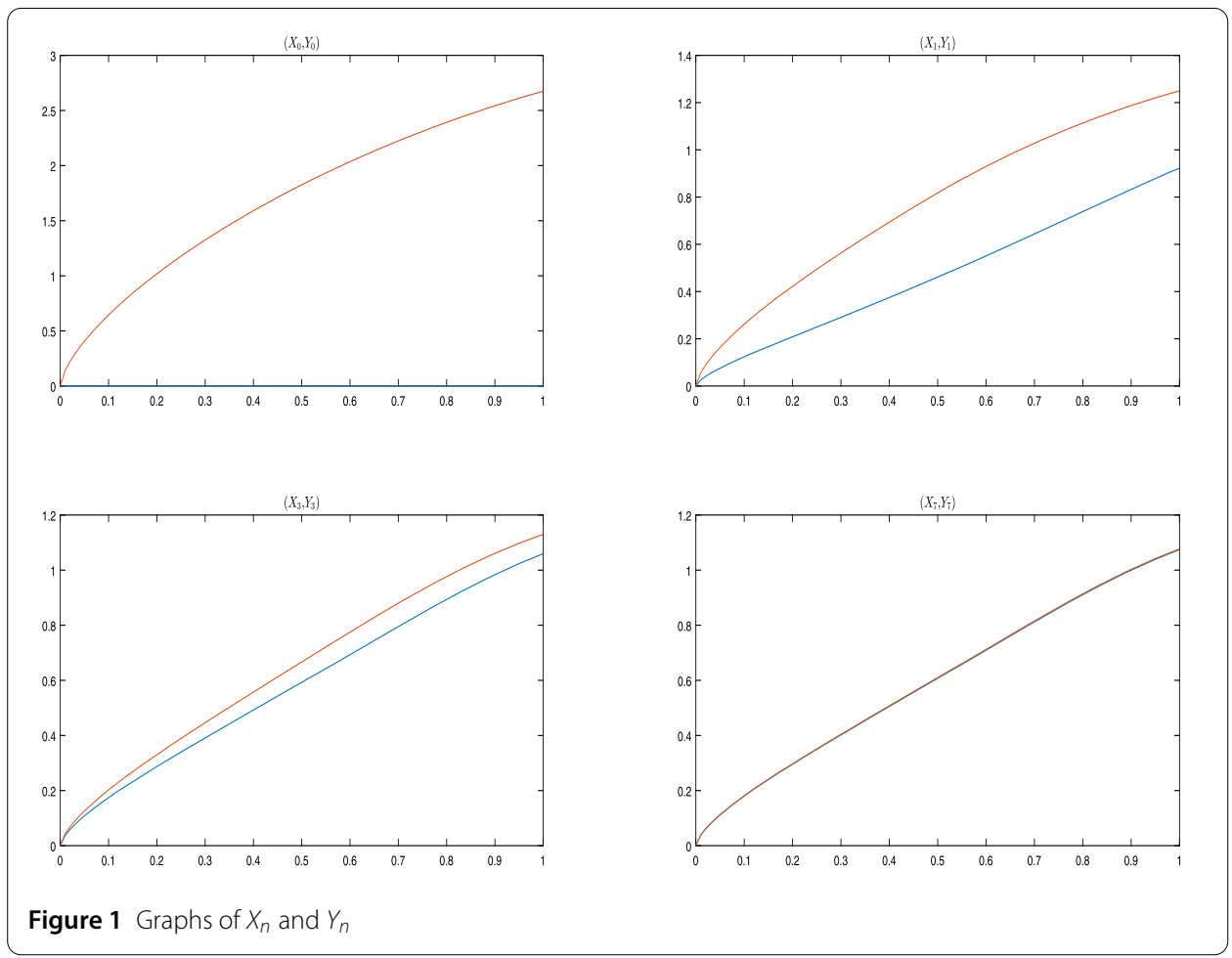

\section{Acknowledgements}

The authors sincerely thanks the editor and reviewers for their valuable suggestions and useful comments to improve the manuscript.

\section{Funding}

This work is supported by Scientific Research Foundation of Hainan Medical University (No. 2020030).

\section{Availability of data and materials}

Data sharing not applicable to this paper as no data sets were generated or analyzed during the current study.

\section{Competing interests}

The authors declare that they have no competing interests.

\section{Authors' contributions}

The authors have made the same contribution. Both authors read and approved the final manuscript.

\section{Author details}

${ }^{1}$ School of Public Health, Hainan Medical College, Haikou 571101, P.R. China. ${ }^{2}$ School of Mathematics and Statistics, Northeast Petroleum University, Daqing 163318, P.R. China.

\section{Publisher's Note}

Springer Nature remains neutral with regard to jurisdictional claims in published maps and institutional affiliations.

Received: 13 October 2020 Accepted: 26 December 2020 Published online: 10 February 2021

\section{References}

1. Zhang, X., Liu, L., Wiwatannapataphee, B., Wu, Y.: The eigenvalue for a class of singular p-Laplacian fractional differential equations involving the Riemann-Stieltjes integral boundary condition. Appl. Math. Comput. 235 412-422 (2014)

2. Lu, H., Han, Z., Sun, S.: Multiplicity of positive solutions for Sturm-Liouville boundary value problems of fractional differential equations with p-Laplacian. Bound. Value Probl. 26, 1-17 (2014) 
3. Li, S., Zhang, X., Wu, Y., Caccetta, L.: Extremal solutions for p-Laplacian differential systems via iterative computation. Appl. Math. Lett. 26, 1151-1158 (2013)

4. Jiang, W.: Solvability of fractional differential equations with $p$-Laplacian at resonance. Appl. Math. Comput. 260, 48-56 (2015)

5. Zhang, X., Liu, L., Wu, Y., Lu, Y.: The iterative solutions for nonlinear factional differential equation. Appl. Math. Comput. 219, 4680-4691 (2013)

6. Zhang, X., Han, Y.: Existence and uniqueness of positive solutions for higher order nonlocal fractional differential equations. Appl. Math. Lett. 25, 555-560 (2012)

7. Li, Y., Lin, S.: Positive solutions for the nonlinear Hadamard type fractional differential equations with p-Laplacian. J. Funct. Spaces Appl. 2013, Art. 951643 (2013)

8. Han, Z., Lu, H., Zhang, C.: Positive solutions for eigenvalue problems for fractional differential equation with generalized p-Laplacian. Appl. Math. Comput. 257, 526-536 (2015)

9. He, Y.: Existence results and the monotone iterative technique for nonlinear fractional differential systems involving fractional integral boundary conditions. Adv. Differ. Equ. 2017, 264 (2017)

10. He, Y., Bi, B.: Existence and iteration of positive solution for fractional integral boundary value problems with p-Laplacian operator. Adv. Differ. Equ. 2019, 415 (2019)

11. Wang, G.: Explicit iteration and unbounded solutions for fractional integral boundary value problem on an infinite interval. Appl. Math. Lett. 47, 1-7 (2015)

\section{Submit your manuscript to a SpringerOpen ${ }^{\circ}$ journal and benefit from:}

- Convenient online submission

- Rigorous peer review

Open access: articles freely available online

High visibility within the field

- Retaining the copyright to your article

Submit your next manuscript at $\gg$ springeropen.com 